\title{
Research on fault feature extraction method of abnormal noise of diesel engine based on wavelet energy spectrum
}

\author{
Bin Zhou ${ }^{1, \mathrm{a}}$, Hui-Juan Feng ${ }^{2, \mathrm{~b}}$ and Shi-Jiu Jin ${ }^{1, \mathrm{c}}$ \\ ${ }^{I}$ College of Precision Instrument and Opto-Electronic Engineering, \\ Tianjin University, Tianjin 300072, China \\ ${ }^{2}$ Department of Automobile Engineering, Tianjin Transportation University, \\ Tianjin,300161,China

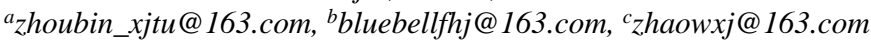 \\ Hui-Juan Feng
}

\begin{abstract}
A data acquisition system has been designed to capture the vibration signal for the engines. A test has been carried out for the vibration signal acquisition of piston knock and piston pin knock of 5.9L Cummins 6BT diesel engine. The test data has been processed by continuous wavelet transform. The scale-energy spectrum and the 3-dimentinal (3D) energy spectrum plot have been generated. The fault feature of abnormal noise of piston knock and piston pin knock of the engine under various technical condition and operating status has been extracted. The research result shows that, the setup of scale-energy spectrum and 3D energy spectrum plot by applying continuous wavelet transform is an efficient method for multi-source abnormal noise extraction for diesel engines, it can not only differentiate piston knock and piston pin knock visually and efficiently, but also can provide useful information to identity the fault level of them.
\end{abstract}

Keywords: Continuous wavelet transform, Diesel engine; Energy spectrum; Fault diagnosis

\section{Introduction}

\section{Background}

With the transform of maintenance approaches from reactive to proactive, there are some new concepts which had been brought into design and maintenance area, such as condition based maintenance. These methods and concepts present large challenge for us to improve the technology and process to meet the new requirements, especially in the field of machine condition monitoring and fault diagnostics. In which, the troubleshooting of abnormal noise of the diesel engines is a hard work for both operators and maintainers. How to improve the accurate and speed of fault locating and isolation is an important problem we have to resolve. The abnormal noise of the diesel engine may come from the crank axle 
and connecting rod bearings, pistons, and piston pin. Among the abnormal noises of a diesel engine, knocks is the most common phenomenon. There are too many causes may result in knocks, such as excessive carbon deposits, scored piston or cylinder, and loose valve set screws [1].

\section{Current researches and problems}

In recent ten years, lots of researches have been carried out for engine fault diagnosis and fault feature extraction by applying wavelet transform and related techniques and methods. E.g., the wavelet packet technique was introduced to deal with the unstable dynamic properties of diesel engines, and the wavelet packet frequency-shifting algorithm was given [2]. An exact wavelet analysis has been designed to enhance the robustness of vibration-based machine fault diagnosis [3]. Discrete wavelet transform technique has been used to decompose the signal into approximations and details for piston cylinder knocking and crank bearing knock [4], etc.

These researches have been greatly propelled the progress of engine fault monitoring and diagnosis. But the problem of engine mechanical fault diagnosis has not been settled up thoroughly. Up to present, most researches are focused on the problem of a single fault (bearing, piston, piston pin, crankshaft, etc.) identification and diagnosis, how to resolve the diagnosis and identification of multi faults which actually occurred in a diesel engine has not been resolved yet.

\section{Goals of research}

The objective of this paper is to develop the technique and method to deal with knock signals from multi components and multi faults of a diesel engine, so as to set up a more precise and feasible method of fault feature extraction and diagnosis for pistons of diesel engine.

Basic Theory

\section{Continuous wavelet transform (CWT)}

The wavelet transform of a continuous signal with respect to the wavelet function is defined as [5]

$$
T(a, b)=w(a) \int_{0}^{\infty} x(t) \psi^{*}\left(\frac{t-b}{a}\right) d t
$$

Where, $w(a)$ is weighting function.$x(t)$ is the signal to be transformed, it could be a beating heart, an audio signal, gearbox vibration, a financial index or peretap $b$ even a spatial signal such as a crack profile or land surface heights. $a$ is the transform wave, and the asterisk indicates that the complex 
conjugate of the wavelet function is used in the transform . $a$ is dilation parameter (scale parameter), which governg the dilation and contraction of the wavelet.$b$ is translation parameter, which govens the movement of the wavelet along the time axis. Typically $w(a)$ is set to $\sqrt{a}$ for reason of energy conservation, thus the wavelet transform is written as

$$
\begin{aligned}
& T(a, b)=\int_{-\infty}^{+\infty} x(t) \psi_{a, b}^{*}(t) d t=<x, \psi_{a, b}> \\
& \text { Where, }{ }^{\psi_{a, b}}(t) \text { is the normalized wavelet function }
\end{aligned}
$$

$$
\psi_{a, b}(t)=\frac{1}{\sqrt{a}} \psi\left(\frac{t-b}{a}\right)
$$

The total energy contained in a signal $x(t)$ is defined as its integrated squared magnitude

$$
E=\int_{-\infty}^{+\infty}|x(t)|^{2} d t=\|x(t)\|^{2}
$$

The relative contribution of signal energy contained at a specific a scale and $b$ location is given by the two-dimensional wavelet energy density function

$$
E(a, b)=|T(a, b)|^{2}
$$

The relative contribution to the total energy contained within the signal at a specific a scale is given by the scale dependent energy distribution

$$
E(a)=\int_{-\infty}^{+\infty}|T(a, b)|^{2} d b
$$

The maximum peak amplitude of energy at a specific scale parameter can be calculated from (6).

\section{The mother wavelet}

It is the most important work to search or build an appreciate function which can be used as the mother wave when we charge various complex signals with wavelet transform. Apparently, select an optimism mother wavelet for multi fault feature extraction is not easy. Fortunately, some researchers have carried out many works for us. In the research conclusion from Xi, an Jiaotong University[6], Morlet wavelet is the best mother wavelet for the feature extraction of shock type machine vibration signals. The vibration signal from a diesel engine is just a shock signal. So it is selected in this paper. 
Morlet wavelet is the most common used complex wavelet, which is defined as[5]

$$
\psi(t)=\frac{1}{\pi^{1 / 4}} e^{i 2 \pi f_{0} t} e^{-\frac{1}{2} t^{2}}
$$

$\psi\left(\frac{t-b}{a}\right)=\frac{1}{\pi^{1 / 4}} e^{i 2 \pi f_{0}[(t-a) / b]} e^{-\frac{1}{2}[(t-a) / b]^{2}}$

\section{Calculation method}

If we have a discrete experimental signal collected, which have been acquired at discrete time intervals from a continuous signal $x(t)$, is of finite length $N$, then the wavelet transform of it can be calculated by[7]

$$
T(a, b)=\frac{\Delta t}{\sqrt{a}} \sum_{i=0}^{N-1} x\left(t_{i}\right) \psi_{a, b}\left(t_{i}\right)
$$

Where, $N$ is the sample numbers. $\Delta t$ is the sample interval. ${ }^{t}$ is the sample time, $t_{i}=i \Delta t, i=0,1, \ldots, N-1 . x\left(t_{i}\right)$ is the sample signal at $t_{i}$.Then

$$
T\left(a_{j}, b_{k}\right)=\frac{\Delta t}{\sqrt{a_{j}}} \sum_{i=o}^{N-1} x\left(t_{i}\right) \psi_{a_{j}, b_{k}}\left(t_{i}\right)
$$

The relative contribution to the total energy contained within the signal at a specific a scale can be calculated as

$$
E\left(a_{j}\right)=\frac{1}{N} \sum_{k=0}^{N-1}\left[T\left(a_{j}, b_{k}\right)\right]^{2}
$$

In this paper, during the calculation, set $a_{j}=2^{j}$, and $b_{k}=2^{j} k, j, k=0,1, \ldots, N-1$.

A continuous wavelet transform coefficient matrix can be gained through the changing of dilation parameter $a$, and $N$. The result can be expressed by a 3D figure which can be called 3D energy spectrum of the wavelet transform coefficient. In this paper, it will be simply written as 3D energy. It can express the changing of $|T(a, b)|$ and $E(a)$ with the time and the scale parameter $a$. 


\section{Fault Feature Extraction}

\section{The design of data acquisition system}

A data acquisition system has been designed to capture the vibration signal of the engine, as shown in Figure 1.

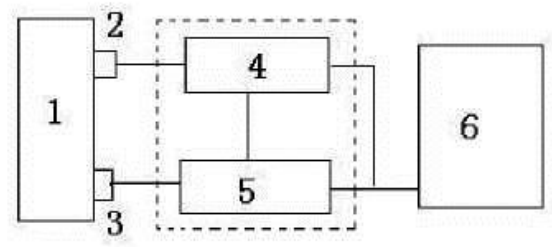

Fig.1Logic block diagrams of data acquisition system

In Figure, 1 represents the engine, 2 represents speed sensor, 3 represents vibration signal sensors, 4 represents speed monitoring device(SMD), 5 represents A/D converter, and 6 represents a computer. A 5.9L Cummins 6BT diesel engine, has been selected as equipment under test and installed on the test bench. Five vibration sensors has been installed to the external of cylinder block respectively to collect vibration signal. The location of each sensor is shown in Table 1.

Table 1Location of the sensors

\begin{tabular}{cll}
\hline No & \multicolumn{1}{c}{ Location } \\
\hline 1\# & 3rd cylinder, external of cylinder block & Top-right \\
2\# & Intermediate between 3rd and 4th main bearing, & Top-left \\
3\# & Right \\
4\# & Right to 3rd cylinder, external of engine oil pan & Left \\
5\# & Bottom \\
\hline \multicolumn{3}{c}{ Note: Cylinder No. is numbered from the front of the engine. }
\end{tabular}

\section{The test data}

During the test, the engine is operating in unloaded condition. Five technique statuses have been set for testing, as shown in Table 2.

Table 2 The technical status of test engine

\begin{tabular}{clll}
\hline No. & Technical status & $\mathrm{PCC}^{1} \mathrm{~mm}$ & $\mathrm{PPC}^{2} \mathrm{~mm}$ \\
\hline A Normal & 0.08 & 0.01 \\
B Piston pin knock(minor) & 0.08 & 0.10 \\
C Piston pin knock(critical) & 0.08 & 0.20 \\
D Piston knock(minor) & 0.20 & 0.01 \\
E Piston knock(critical) & 0.40 & 0.01 \\
\hline
\end{tabular}

${ }^{1}$ Piston-cylinder clearance. 
${ }^{2}$ Piston-pinhole clearance.

Four operating speeds, i.e., 800 (Idle speed), 1300 (low speed), 1800 (moderate speed), and 2300 (moderate-high speed) r/min, has been selected for data acquisition.Sampling frequency is $25.6 \mathrm{kHz}$, and sampling point numbers is 4096. Twenty groups of data under various technique statuses at different operating speed has been acquired.

\section{Scale-energy spectrum analysis}

Taking Morlet wavelet as the mother wavelet, applying CWT for each data, the wavelet transform coefficient matrix has been gained. Then equation (9) and (10) can be used to calculate the energy. And the energy spectrum of each speed and each technical status can be drawn up.

And the square of coefs is the energy. The maximum peak amplitude of energy/Scale parameter under each technical status and operating speed from each measuring location are shown in Table 3.

Table 3The maximum peak amplitude of energy scale parameter Speed Maximum peak amplitude of energy Scale parameter

\begin{tabular}{cllllll} 
TS & r/ & $1 \#$ & 2\# & $3 \#$ & $4 \#$ & 5\# \\
\hline A & 80 & 0.1699 & $0.10425 /$ & 0.3305 & $0.20371 /$ & 0.24224 \\
& 13 & 0.6985 & $0.1373 / 9$ & 0.8856 & $0.58775 /$ & 0.87182 \\
& 18 & $1.4496 /$ & $0.27782 /$ & 0.9667 & $1.0018 / 1$ & 0.91766 \\
& 23 & $5.9588 /$ & $1.3098 / 8$ & $3.3681 /$ & $2.3045 / 6$ & $2.4483 /$ \\
B & 80 & 0.1191 & $0.11438 /$ & 0.4873 & $0.23827 /$ & 0.21101 \\
& 13 & $1.4823 /$ & $0.29192 /$ & $1.4606 /$ & $1.0699 / 1$ & $0.9939 /$ \\
& 18 & $1.462 / 6$ & $1.4019 / 7$ & $2.9088 /$ & $1.4031 / 1$ & $1.4833 /$ \\
& 23 & $3.7883 /$ & $2.3137 / 7$ & 13.705 & $3.0394 / 6$ & $7.6125 /$ \\
C & 80 & 0.1648 & 0.07998 & $0.1345 /$ & $0.17145 /$ & 0.37656 \\
& 13 & 0.8721 & $0.51944 /$ & 0.9679 & $0.71208 /$ & $1.4926 /$ \\
& 18 & $2.0311 /$ & $4.1526 / 5$ & $5.1899 /$ & $1.9228 / 1$ & $3.0783 /$ \\
& 23 & $3.578 / 7$ & $4.9182 / 5$ & $3.4052 /$ & $4.2522 / 1$ & $9.0185 /$ \\
$\mathrm{D}$ & 80 & $0.2164 /$ & $0.32353 /$ & 0.3408 & $0.24324 /$ & 0.36183 \\
& 13 & $1.828 / 5$ & $2.0558 / 6$ & $1.9732 /$ & $0.59601 /$ & $1.2827 /$ \\
& 18 & $2.5736 /$ & $2.3935 / 6$ & $3.4119 /$ & $1.3965 / 5$ & $2.5408 /$ \\
& 23 & $8.3495 /$ & $5.9513 / 5$ & $9.4273 /$ & $2.6787 / 6$ & $6.962 / 7$ \\
E & 80 & $0.2206 /$ & $1.5873 / 4$ & 0.7370 & $0.33868 /$ & 0.56001 \\
& 13 & $2.273 / 5$ & $5.331 / 5$ & $4.1305 /$ & $1.0377 / 5$ & $2.1577 /$ \\
& 18 & $3.7998 /$ & $12.4565 /$ & 12.681 & $1.9764 / 6$ & $7.1746 /$ \\
& 23 & $6.5016 /$ & $16.4506 /$ & 34.409 & $4.396 / 7$ & 13.8835 \\
\hline TS: Tech & & &
\end{tabular}

TS: Technical status

From Table 3, some conclusion can be drawn out: 
(1)At the top-left of cylinder block and right of crankshaft main bearing, the range of scale parameter corresponding to the maximum amplitude is concentrated to 4-10.

(2)When the scale parameter is about 4-10, at the top-left of cylinder block and the right of crankshaft main bearing, the maximum amplitude is increasing with the operating speed.

(3)When scale parameter is about 4-10, and the operating speed is 1300 and 2300r/min, at the top-left of cylinder block, the maximum amplitude is increasing with the fault level at a fixed proportionality. And the different of the corresponding maxi-mum amplitude of the two faults is rather large, especially at 1300r/min,. Thus, the characteristics of the maximum amplitude at the top-left of cylinder block is the most significant at 1300 and 2300r/min, which is shown in Figure 2.

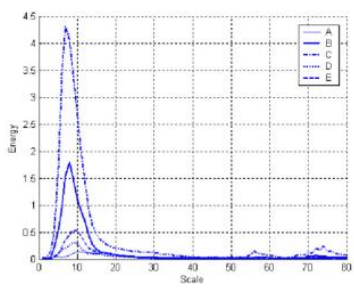

(a)

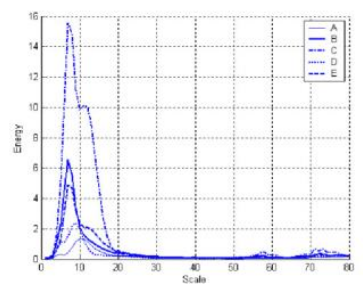

(b)

Fig.2Scale-energy spectrum:(a) 1300r/min; (b) 2300r/min

According to the analysis above, when the engine is operating at $1300 \mathrm{r} / \mathrm{min}$, when the scale parameter is about 4-10, the vibration signal from the top-left of cylinder block can distinguish the piston knock and piston pin knock and its fault level after the Morlet wavelet transform by the maximum peak amplitude of energy.

Apparently, the calculating results can be further processed for other usages. For example, least square method can be applied to draw a clearance-energy plot, see Figure 3. And the fit clearance between piston and cylinder, the piston pin and pinhole can also be reckoned. In Figure 3, curve 1 is the fit clearance between piston and cylinder, and curve 2 is the fit clearance between piston pin and pinhole. 


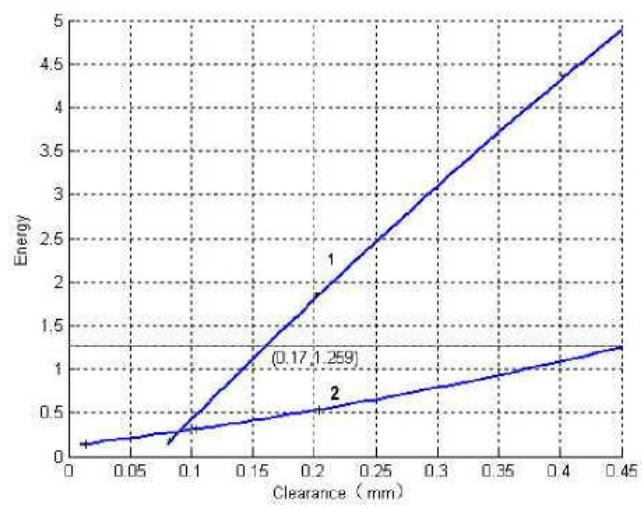

Fig.3Clearance-energy plot

In practical maintenance actions of diesel engine, the piston knock and piston pin knock is generally distinguished by experiences. When the engine is operating at idle or higher speed, the piston knock is clear. When the engine is operating at idle speed higher, the piston pin knock fault will be clear[8]. That is to say that, the analysis conclusions by scale-power spectrum is consisting with actual maintenance experiences very well.

\section{D Energy Spectrum Analysis}

As discussed above, scale-energy spectrum can be applied to differentiate piston knock and pin knock and the fault levels very well. But there are some disadvantages of this method. For example, the range of the scale characteristics of the two kinds of fault is nearly the same. From Figure 2, when the energy is less than 0.6411 , these two faults can be differentiate by scale-energy spectrum. So, 3D energy spectrum analysis has been carried out to make up the disadvantages. The 3D energy spectrum plots of the engine are shown in Figure 4 and Figure 5.

In Figure 4 and Figure 5, the sample position is at 3rd cylinder, external position of cylinder block top-left, the scale parameter is 3-20.

From figures, the wavelet power is increasing with the engine speed and fit clearance, and it seems more apparent when the 3rd cylinder (fault cylinder) is outputting capacity. And the changing of the fit clearance between the piston and cylinder can affect the wavelet power mostly.

(1) When the 3rd and 4th piston are at the top dead center nearby, the wavelet power is increasing with the fit clearance between the piston and cylinder significantly. That means the shock signal (hereby will simply written as shock) of the engine is obvious. With the fit clearance increases, when the engine speed is $1300 \mathrm{r} / \mathrm{min}$, the shock is focus on the top dead center of the 1 st nearby except the 
$3 \mathrm{rd}$ and 4 th piston. But, when the engine speed is $2300 \mathrm{r} / \mathrm{min}$, the shock is focusing on the top dead center of the 3rd, 6th, 2nd, and 4thnearby. And, with the operating speed increasing, the shock is more distinct. This is just fit with the operating characteristics of the engine, whose power order is 1-5-3-6-2-4, and when the 3rd and 4 th cylinder are at top dead center nearby, the 3rd piston is just at the top dead center nearby, and the shock is increasing with the fit clearance.

(2) When the fit clearance between piston pin and pinhole is smaller $(0.10 \mathrm{~mm})$, a larger shock will be observed from each cylinder at top dead center position at the speed of $1300 \mathrm{r} / \mathrm{min}$. If the speed is changed to $2300 \mathrm{r} / \mathrm{min}$, the shock from the 3rd and 6th cylinder is larger when they are at top dead center. When the fit clearance is changing to larger $(0.20 \mathrm{~mm})$, the shock characteristic is just changed at $1300 \mathrm{r} / \mathrm{min}$, and the shock from $1 \mathrm{st}$, 5th and 3rd cylinder is larger at top dead center. From the analysis above, there are obvious differences between the two faults at the 3D energy spectrum plot. The fault characteristic of piston knock is distinct at $1300 \mathrm{r} / \mathrm{min}$ when the fit clearance between the piston and cylinder is increasing. The fault characteristic of piston pin knock is distinct at 2300r/min when the fit clearance between the pistons pin and pinhole is increasing.

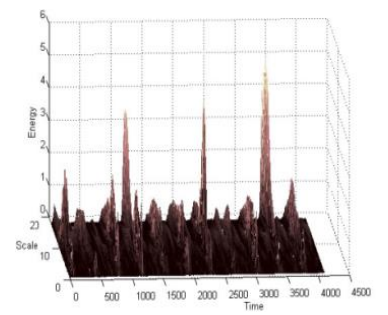

(a)

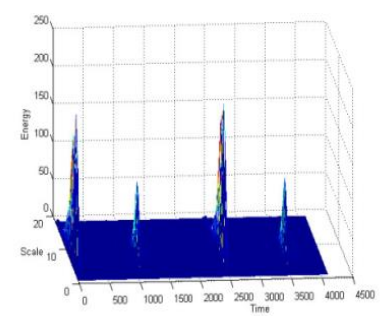

(b)

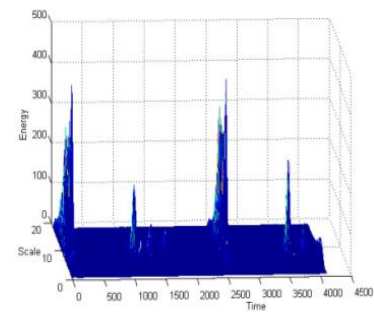

(c)

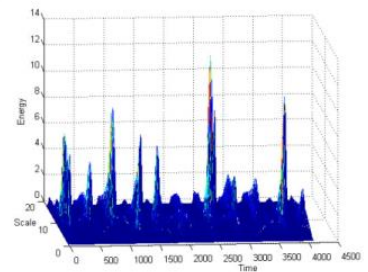

(d)

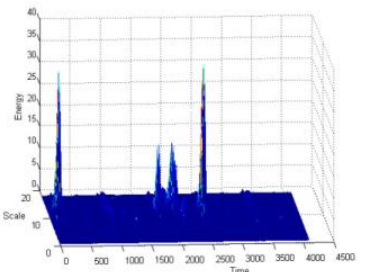

(e)

Fig.43D energy spectrum plot, 1300r/min

(a) Normal; (b)PCC $=0.2 \mathrm{~mm}$; (c) $\mathrm{PCC}=0.4 \mathrm{~mm} ;(\mathrm{d}) \mathrm{PPC}=0.1 \mathrm{~mm}$;(e)PPC $=0.2 \mathrm{~mm}$ 


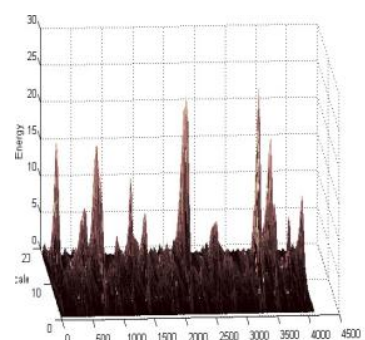

(a)

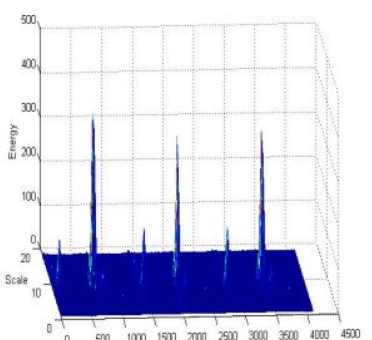

(b)

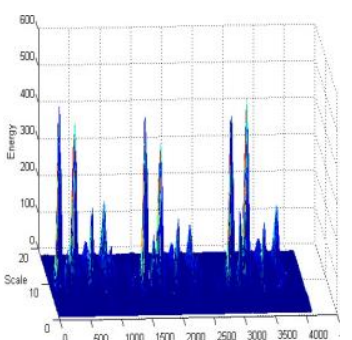

(c)

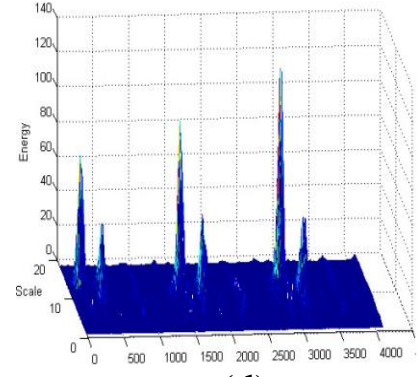

(d)

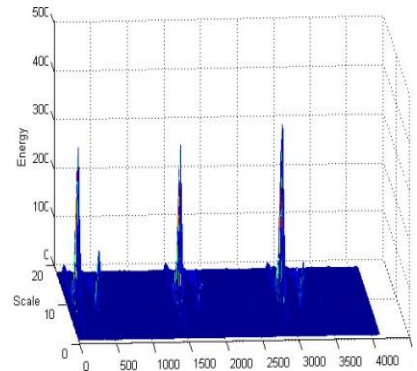

(e)

Fig.53D energy spectrum plot, 2300r/min

(a) Normal; (b) PCC $=0.2 \mathrm{~mm}$; (c)PCC $=0.4 \mathrm{~mm}$; (d)PPC $=0.1 \mathrm{~mm}$; (e) $P P C=0.2 \mathrm{~mm}$

\section{Conclusions}

(1) Both scale-energy spectrum and 3D energy spectrum of Morlet continuous wavelet are suitable to extract fault features from vibration signals of the cylinder block from a Cummins 6BT engine. And 3D energy spectrum is superior to the scale-energy spectrum in differentiating piston knock and piston pin knock as well as their fault levels. If the two methods can be combined together, a more visual, precise, and effective method of fault feature extraction and diagnosis can be obtained.

(2) To detect and differentiate the piston knock and piston pin knock efficiently for Cummins 6BT engine, the vibration signal should be sampled in the position of the top left of the cylinder block and the operating speed be about $1300 \mathrm{r} / \mathrm{min}$ to $2300 \mathrm{r} / \mathrm{min}$, and the optimism analysis scale parameter should be about 4-10.

(3) Through follow-up data process, e.g. the clearance-power plot described in this paper can be used to deduce the fit clearance between the cylinder and piston, and the piston pin and pinhole. And it can be used to identify the technical status of the engine, so as to provide decision basis for condition based maintenance. 


\section{References}

[1] R. K. Mobley, L. R. Higgins, D. J. Wikoff, Maintenance Engineering Handbook, 7th Edition, McGraw-Hill Companies, Inc., NY, 2008, pp.5.194-5.196.

[2] Z. Jian, C. Jin, Z. Jun, et al, Diesel engines' vibroacoustic signature extraction by wavelet packet technique, Journal of Shanghai Jiaotong University vol.E-7, No.1 (2002) pp.95-99.

[3] P. W. Tse, W. xian Yang, H. Tam, Machine fault diagnosis through an effective exact wavelet analysis, Journal of Sound and Vibration vol.277 (2004) 1005 C1024.

[4] Z. Yuping, Z. Dejiang, W. Weizheng, Fault diagnosis of engine abnormal sound based on wavelet transform technique, Journal of Mechanical Engineering vol. 45, No. 6 (2009) 239-245.

[5] P. S. Addison, The Illustrated Wavelet Transform Handbook, Institute of Physics Publishing, London, UK, 2002, 6-64.

[6] 13C. ZeXin, The extracting technique of diagnosis information of rotating machinery, Ph.D. thesis, Xian Jiaotong University, Xian China, 20-55 (2005).

[7] 14Y. Fusheng, The Engineering Analysis and Application of Wavelet Transform, Science Press, Beijing, China, 2006, 12.

[8] 15Z. Shicai, Y. Wancheng, Automobile Maintenance Techniques, Tianjin Transportation University, Tianjin, China, 2003, 392-401. 